\title{
Measurements of the ns-laserpulse induced expansion of (111) silicon below and above the melting threshold on the nanosecond time scale
}

\author{
F. Kneier ${ }^{1}$, T. Geldhauser, P. Leiderer, J. Boneberg \\ University of Konstanz, Fach 767, 78457 Konstanz, Germany, Email: fabian.kneier@uni-konstanz.de, Fax: +49 7531883791
}

\begin{abstract}
The surface displacement of 111-silicon wafers of $0.675 \mathrm{~mm}$ and $3.05 \mathrm{~mm}$ thickness, respectively, during intense ns laser irradiation is determined on the nm-vertical and ns-time scale using an optimized Michelson interferometer. The surface dynamics is observed below as well as above the melting threshold. We show that the obtained surface expansion below the melting threshold is in good agreement with theoretical heat transfer calculations. Additionally, thickness-dependent bending in the samples is illustrated and the acoustic reflections from the backside of the sample are observed. Maximum thermal displacement at the first expansion plateau is around $6 \mathrm{~nm}$ before melting occurs. We show that qualitative agreement between experimental results and simulation above the melting threshold can be established for the first time by taking the phase shift change in the reflection for molten silicon into account.
\end{abstract}

\section{Introduction}

Thermal heating of substrate surfaces or thin film systems by means of intense laser pulses has been studied for a long time. The resulting expansion dynamics on a nanosecond time scale has moved into the focus of research when the mechanisms behind a variety of phenomena needed to be determined. For dry laser cleaning, i.e. using intense laser irradiation to detach small particles from a substrate surface, an analysis of the nanomechanical detachment process was made possible only by directly determining surface accelerations on the ns-time scale in question [1]. The same holds for acoustic laser cleaning [2] with its possibility of larger surface accelerations by means of a travelling acoustic wave to the opposite side of the irradiated area. Here, discrepancies between maximal surface velocity and detached particle velocity could be evidenced. Furthermore, the recent works of Bringa et al. [3] and Wang et al. [4] have shown that hardening of nanocrystalline materials in the bulk is possible through the application of bulk acoustic waves with very high pressure amplitudes. Again, the important pulse shapes could be observable by its corresponding displacement effect on the surface. Thus, the determination of the ns surface dynamics on a quantitative level is of fundamental importance in many nanomechanical applications.

We would like to show here the expansion dynamics of two Si substrates with (111) orientation $(675 \mu \mathrm{m}$ thin and $3.05 \mathrm{~mm}$ thick) on the nanosecond time scale.

\section{Experimental Setup}

The pulsed laser source used in our experiments for the generation of the thermal expansion is a frequency-doubled Nd:YAG laser ( $\lambda=532 \mathrm{~nm}$, FWHM $10 \mathrm{~ns}$ ). The detection setup for the time-resolved measurements of the displacement of the reflecting sample surface consists of a modified Michelson interferometer (Fig. 1), as decribed in [5]. The interferometer laser source is an Arion laser $(\lambda=488 \mathrm{~nm}, 300 \mathrm{~mW}$ cw output, single longitudinal mode). Polarization optics are utilized in order to gain the full information. The beam is split at a polarizing beam splitter cube, quarter-wave plates in the reference and sample arms rotate the polarization after two passes and achieve an output from both arms entirely to the detectors. A second polarizing beam splitter cube brings the orthogonally polarized beams into interference. Signal B is the inverse interference pattern of A. Advantages of the polarizing optics include minimizing reflections from beam splitter surfaces, prevention of back-reflection of laser light into the laser, easy control of different reflectivities of sample surfaces as well as, most significantly, the ability to use the full information of the interference pattern, namely the inverse signal as well. A balanced setup is realized with two detectors. Both measure the intensity of the center portion of the circular interference pattern. An active stabilization is 


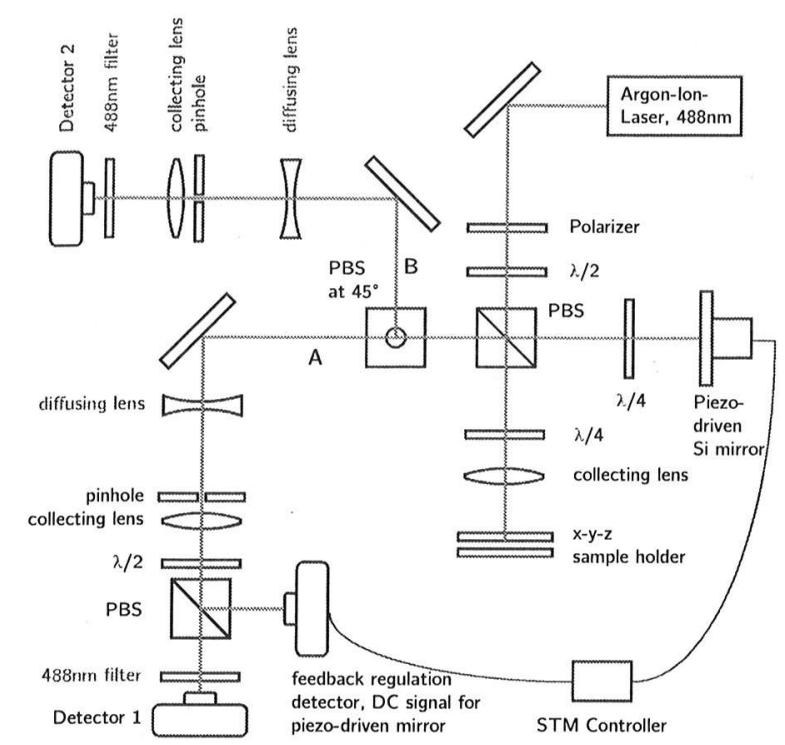

Fig. 1 Schematic setup of the modified Michelson interferometer.

provided through a piezoelectrically driven silicon mirror in the reference arm by means of a feedback loop utilizing a common STM control unit. This allows to set the reference point at the start of the experiment and to operate in the most sensitive, i.e. linear regime of the interference signal around the point exactly in between completely destructive and maximally constructive interference. The sample is irradiated with the Nd:YAG pulse on the interferometer side. The displacement $u$ is acquired from the center intensities at the two detectors using the sum $S=\frac{1}{2}\left(I_{A}+I_{B}\right)$ and the difference $D=\frac{1}{2}\left(I_{A}-I_{B}\right)$. The change in reflectivity $X=\frac{R(t)}{R_{0}}$ of the sample during the irradiation does not need to be obtained in an independent experiment, but instead can be deduced directly, forgoing any uncertainties due to pulse-to-pulse variations

$$
X=2\left(\frac{S}{\frac{1}{2} I_{\max }}-1\right)
$$

The absolute and relative displacements $-l$ and $u$, respectively - are obtained from

$$
\begin{aligned}
l(D) & =\frac{\lambda}{2 \pi} \cdot \arccos \left(\frac{\frac{D}{\frac{1}{2} I_{\max }}}{\left(1+\frac{1}{2} X\right)}\right) \\
u & =l\left(D_{\text {signal }}\right)-l\left(D_{0}\right)
\end{aligned}
$$

with the maximum constructive intensity $I_{\max }$ and the reference point at the start of the experiment $D_{0}$, in general set to zero $\left(I_{A}=\frac{1}{2} I_{\max }=I_{B}\right)$.

The stabilization eliminates slow mechanical vibrations up to $1 \mathrm{kHz}$. Faster signals will not be affected. The used amplifiers are determined to limit gained signals to be faster than $500 \mathrm{kHz}$, thus putting the longtime limit of valid observations at $1 \mu \mathrm{s}$. The time resolution is tested against a fs laser pulse, which yielded the

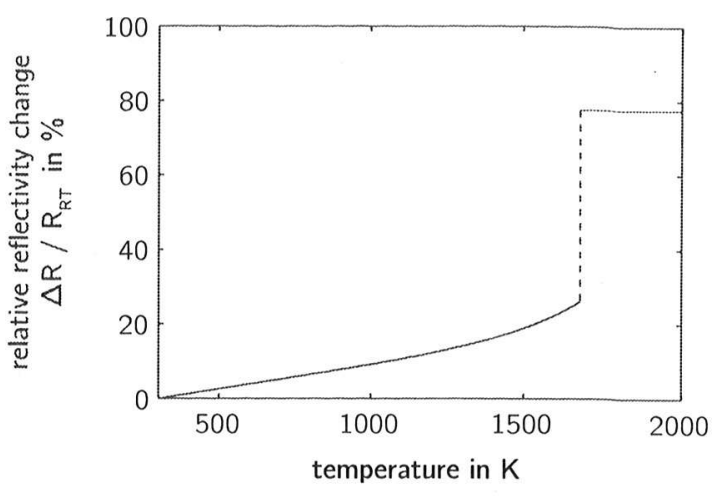

Fig. 2 Temperature dependence of reflectivity of solid and liquid silicon $\Delta R(T)=R(T)-R_{R T}$ with respect to the reflectivity $R_{R T}$ at room temperature.

FWHM of the detectors as 775 ps and 510 ps, respectively. The displacement resolution is $0.55 \mathrm{~nm}$ for single shot and $0.28 \mathrm{~nm}$ for multi-shot experiments. The Arion spot focus on the sample is $100 \mu \mathrm{m}$. The Nd:YAG irradiates the sample at an incidence of $44^{\circ}$ and only slightly focussed, ensuring a larger spot size than the interferometric beam on the sample.

\section{Results and discussion}

\subsection{Reflectivity change due to heating}

When irradiating the sample on the interferometer side the interferometric signal is influenced not only by the thermal expansion, but by the change in the reflectivity of the surface due to the induced heating from the ns-laser pulse as well. This change is determined from the sum of the inverse signals, and incorporated in (2), thus eliminating its effect on the determination of the displacement. Its use as indicator of the phase state of the surface during the experiment is crucial for example when determining the time span during which a different phase shift at the interferometer beam has occurred (see Sect. 3.3). The reflectivity is a result of the electronic band structure and its temperature dependence is given by the temperature dependence of the optical constants $n_{\lambda}(T)$ and $k_{\lambda}(T)$ in the complex refractive index $\hat{n}$

$$
R_{\lambda}(T)=r^{*} \cdot r=\left|\frac{\hat{n}_{0}-\hat{n}_{1}}{\hat{n}_{0}+\hat{n}_{1}}\right|^{2}
$$

From Eq. (4) and with the temperature dependence of the optical constants at $\lambda=488 \mathrm{~nm}$ for crystalline silicon [6-8] and liquid silicon [9] the resulting graph for the reflectivity is calculated in Fig. 2. The relative reflectivity change referenced to room temperature increases up to about $26 \%$ at the melting point. Upon melting a jump towards a theoretical value of $78 \%$ occurs. Experimental results of three fluences in Fig. 3 show that for 236 $\frac{\mathrm{mJ}}{\mathrm{cm}^{2}}$ the $\mathrm{R}$-value is below the melting point maximum. 


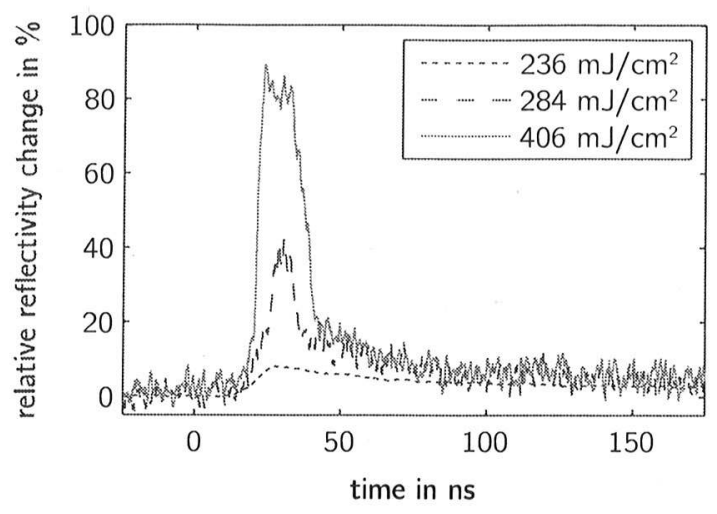

Fig. 3 Relative reflectivity change of 111-silicon at three fluences. Noise at the lowest fluence is less as the signal was averaged over 10 pulses.

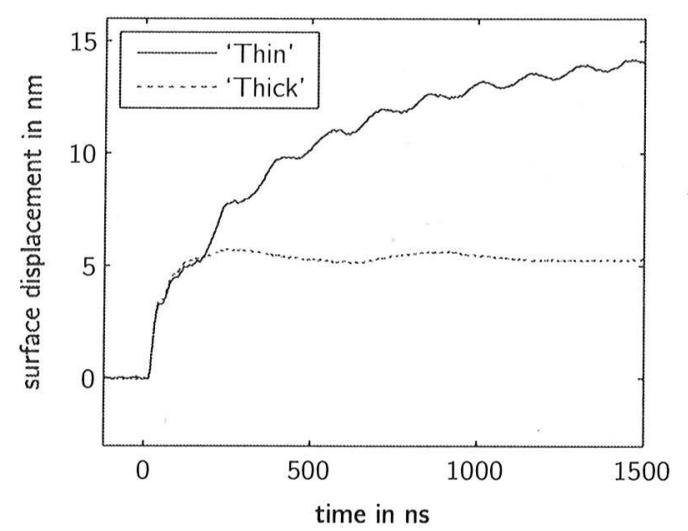

Fig. 4 Comparison of irradiated $0.675 \mathrm{~mm}$ 'thin' and 3.05 mm 'thick' silicon wafer at $236 \frac{\mathrm{mJ}}{\mathrm{cm}^{2}}$ : Displacement signal.

As fluence increases, so does the reflectivity change, and a dip in the expansion (which will be discussed in Sect. 3.3 ) is observed. This correlates well with the R-value being between the maximal crystalline and the liquid value, indicating a partial melting of the silicon thickness probed by the Ar-ion beam and a contraction of the melted material with its $10 \%$ increase in density. At $406 \frac{\mathrm{mJ}}{\mathrm{cm}^{2}}$ a slab of thickness larger than the penetration depth of the Ar-ion laser is being melted during pulse irradiation as seen by the plateau of around $80 \%$.

\subsection{Thermal expansion below the melting point}

The surface displacement of an irradiated $0.675 \mathrm{~mm}$ thin silicon wafer is shown in Fig. 4 ('thin'). After a first expansion period of 20-40 ns a short plateau is reached. Subsequently, further expansion occurs. The signal consists of a continuously increasing background superimposed on which weak undulations occur in regularly spaced time intervals. The background represents a bending of the thin sample under the stress of heating, as a

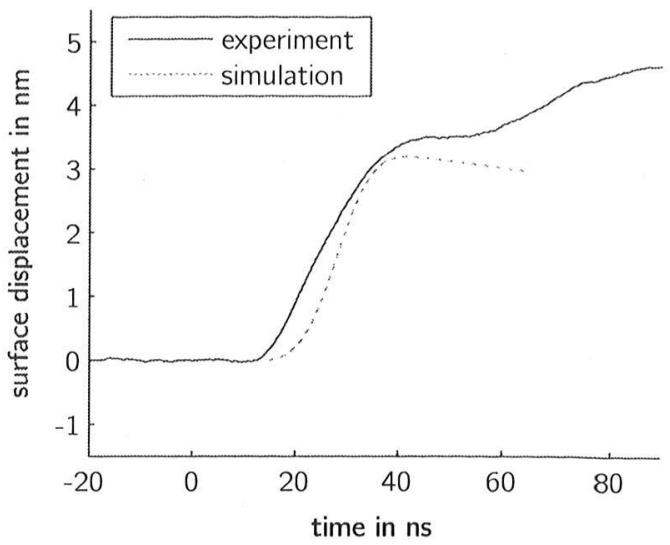

Fig. 5 Comparison of simulated curve and experimental displacement signal for $236 \frac{\mathrm{mJ}}{\mathrm{cm}^{2}}$.

comparison with a more massive $3.05 \mathrm{~mm}$ thick wafer confirms. Its dimensions compared to the molten silicon volume lead to negligible effects of macroscopic bending as evidenced in Fig. 4 ('thick') which shows no continuous increase, but retains weak superimposed undulations of constant time intervals. The difference between the two graphs is an estimate of the bending effect. The superimposed peaks mark the reflections of the first expansion signal which propagates into the bulk silicon as a longitudinal acoustic wave and upon reflection at the backside are registered again by the interferometer. This agrees well with the travel times between the peaks which are around 150 ns for the thin sample and circa 675 ns for the thick wafer, respectively. The sound velocity of the longitudinal acoustic wave in the specific crystal direction is $9.11 \times 10^{3} \frac{\mathrm{m}}{\mathrm{s}}$ [10]. Measurements for the thin wafer yield $(8.83 \pm 0.39) \times 10^{3} \frac{\mathrm{m}}{\mathrm{s}}$, for the thick sample $(9.02 \pm 0.10) \times 10^{3} \frac{\mathrm{m}}{\mathrm{s}}$. The corresponding peak height of reflected displacements is around $0.3 \mathrm{~nm}$.

In order to compare to theoretical predictions numerical simulations are carried out. The temperature distribution for slabs of few $\mathrm{nm}$ thicknesses is obtained in the case of 1-dimensional heat transfer from the ns laser pulse into the substrate. Temperature is related to linear thermal expansion and the theoretical signal is then compared to the experimental result at the corresponding irradiated fluence in Fig. 5. In the simulated data the linear surface expansion takes place within a time frame of 20-30 ns. Signal heights are reproduced well, the small deviation can be attributed to an uncertainty in matching theoretical to experimental fluence. Overall, the simulated data reflect the measured signal well, thus the linear thermal expansion accounts for the first rise in the surface displacement signal. Downward expansion of the heated material is restricted, leading to a deformation in the crystal bonds and therefore to the wave propagating into the substrate that was observed through the reflections above. At the same time the frustrated expansion leads to an additional upward 


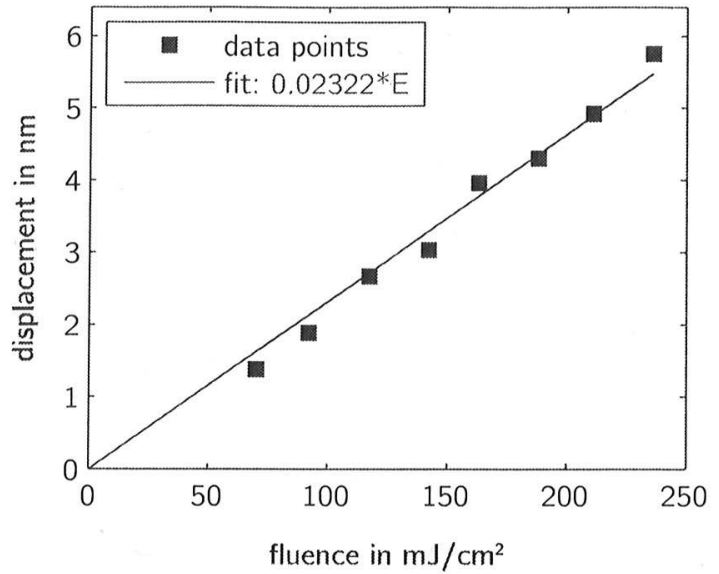

Fig. 6 Surface displacement of silicon irradiated on the interferometer side as a function of different fluences.

displacement with a not separately distinguishable and therefore unknown temporal evolution. The second rise after the thermal plateau starting around the 50-60 ns mark and its maximum at circa $87 \mathrm{~ns}$ fit well into the 150 ns wide reflection peak intervals which follow from circa 236 ns. This suggests that the second peak is associated with the wave propagating into the substrate. A complete understanding would require further investigations as the laterally bounded expansion might also play a role, which might be determinable through systematic studies using different irradiation spot sizes.

Below the melting threshold the thermal expansion of (111) silicon was determined at the thermal plateau after circa $20 \mathrm{~ns}$ as a function of the irradiated fluence. The results are shown in Fig. 6. A first approximation confirms the order of magnitude of the measurements with a thermal diffusion length in silicon over the $20 \mathrm{~ns}$ of $l_{\text {th }}=\sqrt{2 D \tau_{\text {pulse }}} \approx 1900 \mathrm{~nm}$ using $D=\frac{\kappa}{\rho C_{p}}$ with corresponding values [11-13]. Maximal expansion of this simply estimated slab below the melting point is $0.5 \%$ [14]. Using an approximation of $0.3 \%$ over the whole slab yields a displacement of $5.7 \mathrm{~nm}$, thus, almost identical to the measured values. The simulation yields a better estimate of the actual temperature distribution and takes a depth of $5000 \mathrm{~nm}$ into account.

\subsection{Surface displacement around and above the melting point}

Starting at fluences in the vicinity of the melting threshold $\left(300 \frac{\mathrm{mJ}}{\mathrm{cm}^{2}}[15]\right)$ the silicon sample experiences cumulative heating or even surface damage due to the $10 \mathrm{~Hz}$ repetition rate of the Nd:YAG laser changing absorption properties and expansion response. Therefore, single shot experiments were necessary. Applying the two detector subtraction method in this case yields an improved $\mathrm{S} / \mathrm{N}$ ratio by more than the factor of 2 over using

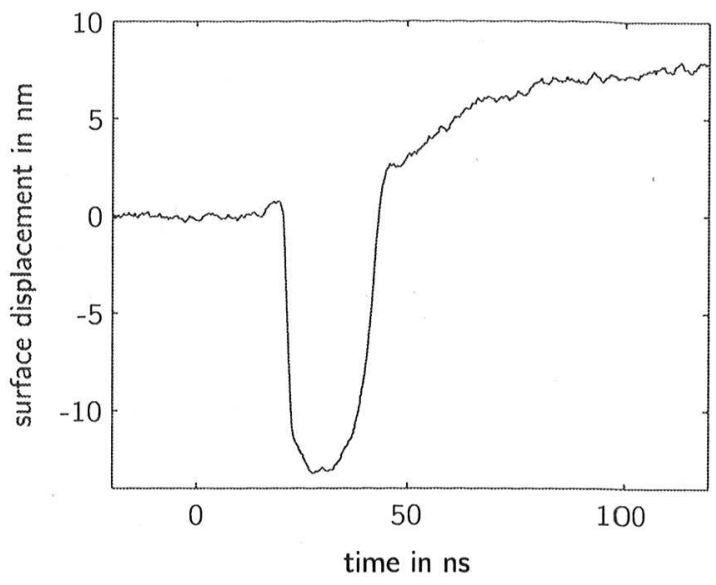

Fig. 7 Surface displacement of silicon for $409 \frac{\mathrm{mJ}}{\mathrm{cm}^{2}}$ without phase shift correction (single pulse irradiation).

one detector only. Fig. 7 shows a typical surface displacement signal for which transient reflectivity change has been incorporated. The occurrence of a dip signifies that part of the sample is molten and the material contracted due to its higher density by about $10 \%$. Estimation of a penetration depth on the order of $30 \mathrm{~nm}$ into the liquid metallic silicon leads to an assumed dip on the order of $3 \mathrm{~nm}$ as opposed to the apparent $15 \mathrm{~nm}$. The reason is found in the changed phase shift at the sample interface for the reflecting interferometer laser when the surface turns from the semiconducting crystalline silicon to the metallic liquid silicon. The phase shift is determined by the generally complex index of refraction. The crystalline interface with its primarily real $\hat{n}$ yields a phase shift of $\pi$ (perpendicular reflection at the optically denser medium) while the liquid interface yields a phase shift corresponding to its non-zero imaginary part in the index of refraction $(\mathrm{n}=2.94, \mathrm{k}=4.99)$. The reflection coefficient for the growing thin film of molten silicon on a crystalline background of the substrate is further complicated by multiple-beam interference. The phase shift is calculated by means of the transfer matrix method as a function of the molten thickness for the Ar-ion wavelength $\lambda=488 \mathrm{~nm}$ and the results shown in Fig. 8. When the sample is molten to a depth equivalent to the penetration depth of the Ar-ion beam, the phase shift changes by $-17.5^{\circ}=-0.305 \mathrm{rad}$. During the time interval that is identified by a plateau in the transient reflectivity change (see Sect. 3.1), a corrected phase shift value needs to be taken into account in the determination of the surface displacement from the interferometric signals. The time the melt front takes to reach this depth is on the order of one to a few nanoseconds as rise and fall times to and from the plateau in the reflectivity change confirm. The exact evolution cannot be taken into account quantitatively, but a qualitative description in which only the transition zones are skipped can be given and is shown in Fig. 9. Fig. 9 (top) illustrates the surface displacement 


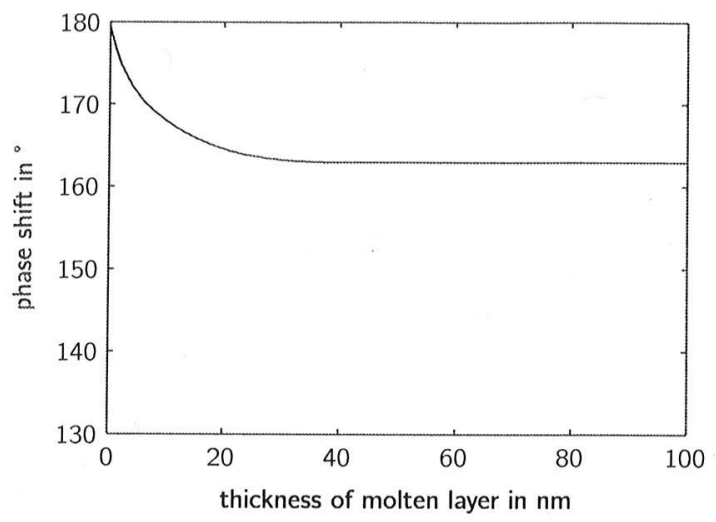

Fig. 8 Phase shift occurring upon reflection of $488 \mathrm{~nm}$ light at system of liquid silicon on crystalline silicon background coming from air. Results are plotted as a function of layer thickness of the molten silicon.
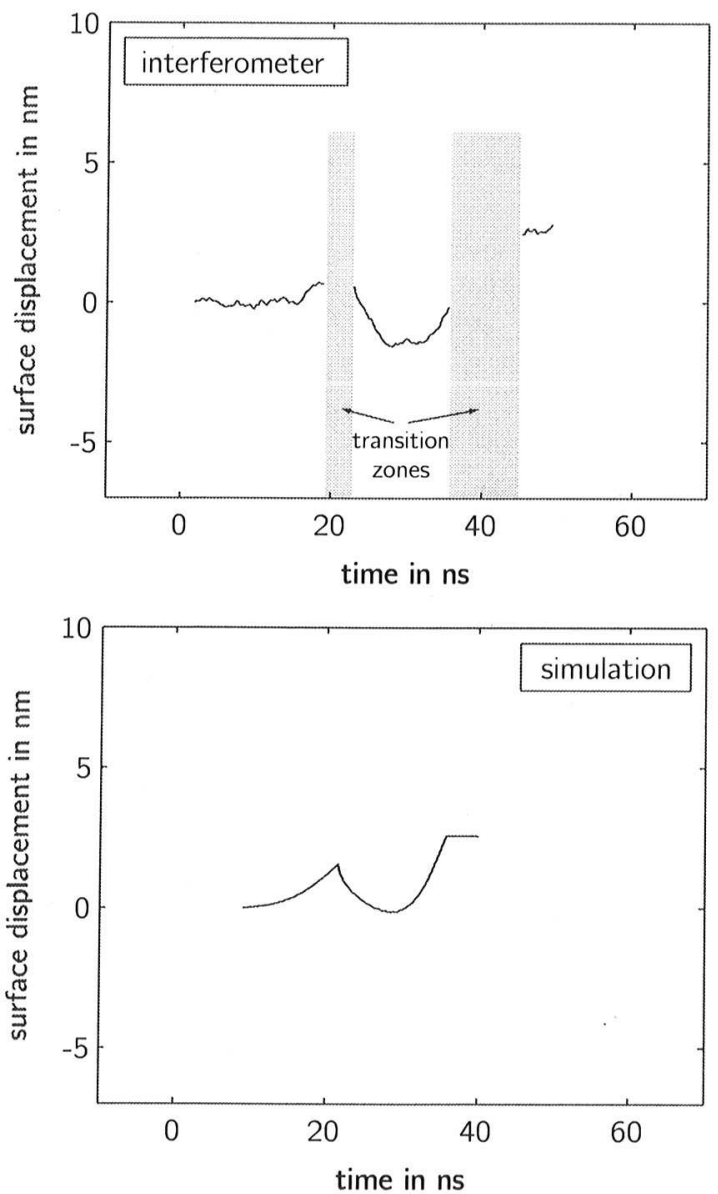

Fig. 9 (top:) Surface displacement of silicon for $409 \frac{\mathrm{mJ}}{\mathrm{cm}^{2}}$ with phase shift correction. During the melting and recrystallization process the exact phase shift cannot be specified, therefore, the data points are left out. (bottom:) Theoretical curve from simulation data. corrected by the changed phase shift without evaluation of the transition zones. The expected alteration to a less pronounced dip is apparent. The matching simulation curve in Fig. 9 (bottom) yields a good qualitative agreement with respect to relative height and width of the depression.

\section{Conclusion}

In conclusion, our experiments show the nanomechanical surface displacement of a silicon sample on the nanosecond time scale in good agreement with theoretical predictions from heat transfer calculations. The thermal expansion of the surface could be demonstrated as well as the phenomenon of bending in thin samples, for which it leads to a strong superposing effect. The relatively simple setup of an optimized Michelson interferometer has yielded a sub-ns and sub-nm resolution allowing to observe even fine details like the occurrence of small peaks corresponding to the reflections of the longitudinal bulk acoustic wave at the backside of the sample on the longer time scale. The surface dynamics was observed at and above the melting threshold of silicon as well. By incorporating the occurrence of a phase shift change during the irradiation, these could be correctly interpreted for the first time and a qualitative agreement with simulation data established. The values obtained for the surface displacement on the nanosecond scale through irradiation with ns-laser pulses provide a fundamental knowledge neccessary in many nanomechanical applications like laser cleaning of silicon substrates or shock hardening of bulk materials.

Acknowledgements We gratefully acknowledge the financial support of the Center of Applied Photonics (CAP) of the University of Konstanz as well as the SFB 767 of the DFG and the Strategic Japanese-German Cooperative Program of the JST and DFG.

\section{References}

1. V. Dobler, R. Oltra, J.P.Boquillon, M. Mosbacher, J. Boneberg, P. Leiderer, Appl. Phys. A 69, (1999) 335

2. T. Geldhauser, F. Ziese, F. Merkt, A. Erbe, J. Boneberg, P. Leiderer, Appl. Phys. A 89, (2007) 109-113

3. E.M. Bringa, A. Caro, Y. Wang, M. Victoria, J.M. McNaney, B.A. Remington, R.F. Smith, B.R. Torralva, H.Van Swygenhoven, Science 309, (2005) 1838

4. Y.M. Wang, E.M. Bringa, J.M. McNaney, M. Victoria, A. Caro, A.M. Hodge, R. Smith, B. Torralva, B.A. Remington, C.A. Schuh, H. Jamarkani, M.A. Meyers, Appl. Phys. Lett. 86, (2006)

5. C.B. Scruby, L.E. Drain, Laser Ultrasonics - techniques and applications (Adam Hilger, Bristol 1990)

6. G.E. Jellison, H.H. Burke, J. Appl. Phys. 60, (1986) 841

7. G.E. Jellison, F.A. Modine, Appl. Phys. Lett. 41, (1982) 180 
8. D.E. Aspnes, A.A. Studna, E. Kinsbron, Phys. Rev. B 29, (1984) 768

9. G.E. Jellison, D.H. Lowndes, Appl. Phys. Lett. 51, (1987) 352

10. O. Madelung, Semiconductors: Data Handbook (Springer, 2002)

11. Y.S. Touloukian, C.Y. Ho, Thermophysical properties of matter - thermal conductivity, Vol. 1: of metallic materials (IFI/Plenum, New York 1970)

12. V.M. Glazov, S.N. Chizhevskaya, N.N. Glagoleva, Liquid semiconductors (Plenum Press, New York 1969)

13. Y.S. Touloukian, C.Y. Ho, Thermophysical properties of matter - specific heat, Vol. 4: of metallic materials (IFI/Plenum, New York 1970)

14. Y.S. Touloukian, R.K. Kirby, E.R. Taylor, T.Y.R. Lee, Thermophysical properties of matter - thermal expansion, Vol. 13: nonmetallic solids (IFI/Plenum, New York 1977)

15. J. Boneberg ( $\mathrm{PhD}$ thesis, konstanzer dissertationen 387, Hartung-Gorre, Konstanz, 1993, ISBN 3-89191-694-9) 